\title{
Revisiting the revised Ag-Pt phase diagram
}

\author{
Gus L. W. Hart ${ }^{a}$, Lance J. Nelson ${ }^{\mathrm{a}}$, Richard R. Vanfleet ${ }^{\mathrm{a}}$, Branton J. Campbell ${ }^{\mathrm{a}}$, Marcel H. F. Sluiter ${ }^{\mathrm{b}}$, \\ Jan H. Neethling ${ }^{c}$, Ezra J. Olivier ${ }^{c}$, Soraya Allies ${ }^{\mathrm{d}}$, Candace I. Lang ${ }^{\mathrm{e}}$, Bryce Meredig ${ }^{\mathrm{f}, \mathrm{g}}$, Christopher \\ Wolverton ${ }^{\mathrm{f}}$ \\ ${ }^{a}$ Department of Physics and Astronomy, Brigham Young University, Provo, Utah 84602, USA \\ ${ }^{b}$ Department of Materials Science and Engineering, Delft University of Technology, Mekelweg 2, 2628 CD Delft, The \\ Netherlands \\ ${ }^{c}$ Centre for HRTEM, Department of Physics, Nelson Mandela Metropolitan University, P.O. Box 77000, Port Elizabeth \\ 6031, South Africa \\ ${ }^{d}$ Centre for Materials Engineering, Department of Mechanical Engineering, University of Cape Town, South Africa \\ ${ }^{e}$ Department of Engineering, Macquarie University, Sydney, Australia. \\ ${ }^{f}$ Department of Materials Science and Engineering, Northwestern University, 2220 Campus Dr., Evanston, USA \\ ${ }^{g}$ Citrine Informatics, 1741 Broadway Street, Redwood City, CA 94063
}

\begin{abstract}
Because of the important applications of platinum alloys and related platinum-group-metals phases, complete phase diagrams for these systems are important for materials engineering. The currently accepted phase diagram for the Ag-Pt system is questionable because of its disagreement with earlier experiments and because of its claim for a lone ordered structure at 53\%-Pt which was not characterized and which contradicts both computational predictions and analogy to the isoelectronic sytem $\mathrm{Cu}-\mathrm{Pt}$. A complete re-examination of the Ag-Pt system by computational and experimental means suggests a phase diagram similar to the isoelectronic system $\mathrm{Cu}-\mathrm{Pt}$. The unknown compound, claimed to be $53 \%-\mathrm{Pt}$, is found to be the $\mathrm{L} 1_{1}$ structure at $50 \%$-Pt.
\end{abstract}

\section{Background}

Platinum alloys and platinum intermetallics play a crucial role in catalytic applications. Because a correct understanding of platinum-based metallic phases is essential to developing new applications and improving existing catalysts, complete compositional phase diagrams for these systems are critical. Unfortunately, many phase diagrams for platinum binary and ternary systems are either incomplete or questionable, due to the uncommon nature of the reported phases. (See discussion in Ref. [16].)

One example is the phase diagram for Ag-Pt, which had undergone few changes since the early work of Schneider and Esch 6, 35, 21 until the mid 1990's. (See the refined, most-widely-accepted phase diagram, until 1996, of Karakaya 20, 25. in Fig. 1). In 1996, Durussel and Feschotte (DF hereafter) gave experimental evidence for a significantly simpler phase diagram, with only one ordered structure - an unidentified structure with a platinum content of $53 \%$. 7] The DF phase diagram, refined by Okamoto 31, is now listed as the pri- mary diagram in the online compilation of phase diagrams (see Fig. 2). 41. (Other references to recent experimental work related to the $\mathrm{Ag}-\mathrm{Pt}$ phase diagram include Refs. [42, 8].)

Based on the early work of Schneider and Esch 35. and subsequent refinements, the phase diagram of DF is surprising. It eliminates two of the intermediate compounds (at $45 \%$ and $75 \%$ platinum), and the $50 \%$-Pt phase, computationallypredicted 3, 27, 38, 33, 28, 4, in several works to be $\mathrm{L}_{1}$ (isostructural to $\mathrm{Cu}_{1} \mathrm{Pt}_{1}$ ), is replaced by a compound at $53 \%$. Based on x-ray diffraction (XRD) and EPMA data, the DF authors speculate that this compound has a concentration of $53 \%$-Pt (atomic \%), with a cubic cell with at least 32 atoms and a stoichiometry of 15:17. [7] But the EPMA method for determining concentrations typically has large error bars (see discussion in Sec. 9p, and their reported XRD data is consistent with the much simpler structure of $\mathrm{Cu}_{1} \mathrm{Pt}_{1}$ (a.k.a. $\mathrm{L}_{1}$ ). $\mathrm{No}_{0}$ $\mathrm{XRD}$ refinement was done in the DF work to determine the structure of the unknown phase. We

October 18, 2016 


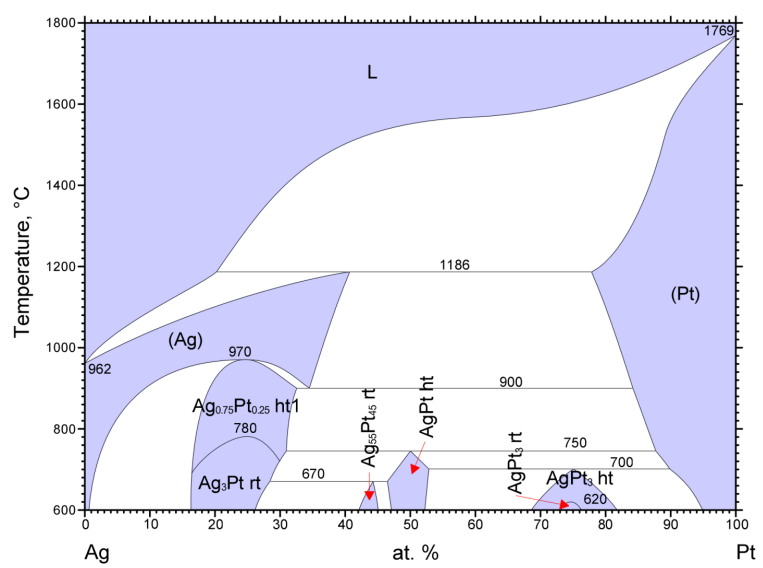

Figure 1: The most widely-accepted phase diagram for the Ag-Pt system (by Karakaya 20 ) prior to the 1996 work of Durussel and Feschotte. 7] Three ordered structures are implied, at $\mathrm{Pt}$ compositions of $45 \%, 50 \%$, and $75 \%$, but no structural data is reported. An ordered phase in the solid solution field is also reported, based on the early work of Schneider 35, originally reported to be $\mathrm{L}_{2}$, (isostructural to $\mathrm{Cu}_{3} \mathrm{Au}$ ).

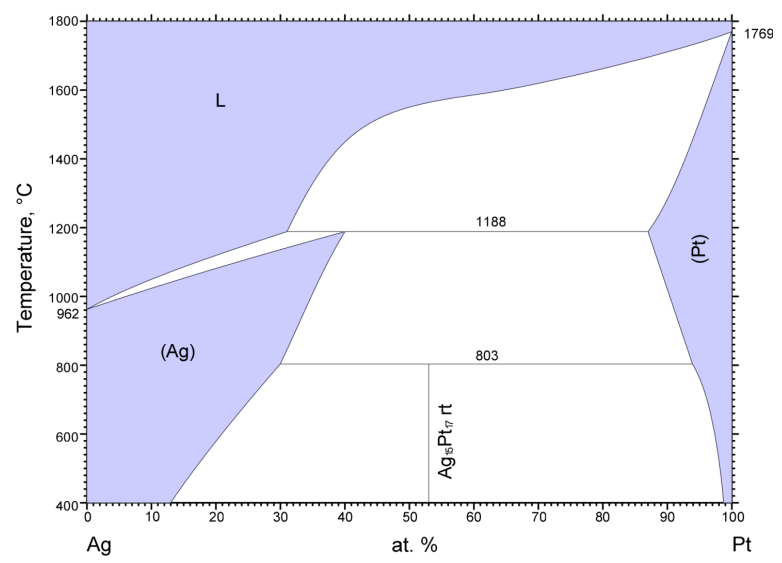

Figure 2: The primary phase diagram for the Ag-Pt system based on the 1996 work of Durussel and Feschotte and redrawn by Okamoto. 31 Only a single compound is reported and no solid solution orderings are given. The structure of the compound at $53 \%$ is not reported but is speculated (based on x-ray diffraction [7]) to be a 32-atom cubic cell with a Ag:Pt stoichiometry of 15:17. are not aware of any other structures with this stoichiometry. It is unfortunate the DF work led to a revision of the phase $\mathrm{Ag}-\mathrm{Pt}$ phase diagram because a reasonable intepretation of the DF work is that the x-ray diffraction data indicated the obvious $\mathrm{L} 1_{1}$ phase at $50 \%$ platinum concentration and that, due to the large error bars, the EPMA-estimated concentration was consistent with this phase.

Several computational studies predict [27, 38, 33, 28, 4] the $\mathrm{L}_{1}$ structure (isostructural to $\mathrm{Cu}_{1} \mathrm{Pt}_{1}$ ), a $50 \%$-Pt compound, in the middle of the phase diagram instead of DF's 53\%-Pt structure. Since ab initio calculations are limited to $T=0 \mathrm{~K}$ predictions and the DF measurements were made at finite temperatures, one is left to wonder whether the discrepancy is genuine or merely due to finite temperature effects. If the differences are real, then we must further ask, "Which results are incorrect? The experimental phase diagram of DF? Or the computational predictions?" One should normally be reluctant to put computational results ahead of experimental ones. However, after a thorough computational examination of the Ag-Pt system, followed by microprobe and TEM studies of samples annealed for months, we claim that the compound proposed by DF is actually $50 \% \mathrm{Pt}$ and is indeed $\mathrm{L} 1_{1}$. This claim is generally consistent with DF's data; it diverges only from their interpretation of their data. In what follows, we chronicle our computational and experimental work that support this claim.

\section{Computational Predictions}

\subsection{Direct DFT Predictions}

Computational studies 27, 38, 33, 28, 4, predict $\mathrm{L1}_{1}$ (isostructural to $\mathrm{CuPt}$ ) to be stable (for $T=0 \mathrm{~K})$ at $50 \% \mathrm{Pt}$ concentration. Recent studies [28, 4, 16] also indicate a stable phase at 40\%-Pt. Figure 3 shows formation enthalpies of 1244 structures calculated via DFT (calculations are described in Ref. 28 ). The structures that were calculated include common alloy phases as well as structures selected from an enumerated list of fcc superstructures. 11, 12, 14, Constructing the convex hull reveals four breaking points, indicating the following stable phases: $\mathrm{L}_{1}(50 \% \mathrm{Pt}), \mathrm{Ca}_{7} \mathrm{Ge}$ $(12.5 \% \mathrm{Pt})$, and two other fcc-derived phases at 3:8 and 3:7 stoichiometry. The 3:7/3:8 stoichiometry phases are $\mathrm{Ag} / \mathrm{Pt}$ stackings in the [111] direction (and thus are similar to $\mathrm{L} 1_{1}$ which is a stacking of 


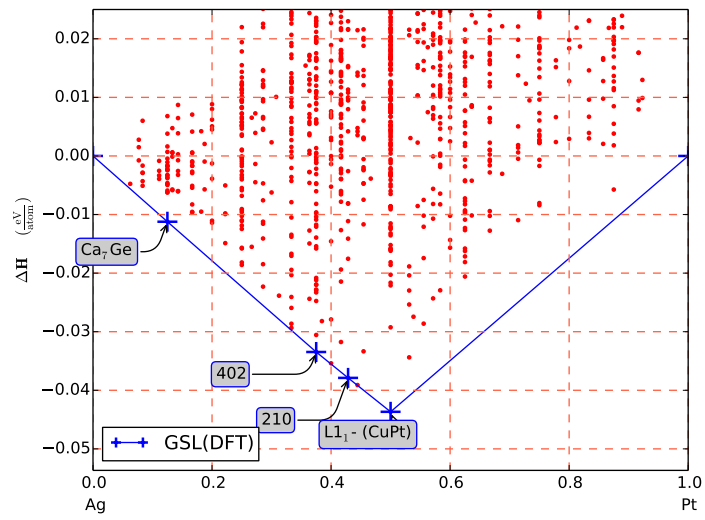

Figure 3: Formation enthalpies of candidate Ag-Pt compounds for 1244 structures calculated via DFT. The convex hull construction indicates four stable phases at low temperatures: $\mathrm{L} 1_{1}, \mathrm{Ca}_{7} \mathrm{Ge}$, and two structures with [111] stacking sequences.

alternating [111] planes of $\mathrm{Ag}$ and $\mathrm{Pt}$ ). A similar $(T=0 \mathrm{~K})$ phase diagram is listed in the AFLOW library. 4

This direct approach, 5, 17] comparing the formation enthalpies of all probable phases, nearly always yields all experimentally-known compounds and often predicts new structures (see, for example, Ref. 16]). On the other hand, it can only make predictions for structures which are included in the calculations - it can make no claim for structures not included in the list; in particular, it does not rule out a hypothetical 32-atom structure with 15:17 stoichiometry, as conjectured by DF. For this, a more thorough search is needed.

\subsection{Cluster Expansion-based Search}

To go beyond this direct approach, one can construct a fast Hamiltonian, which can then be used to conduct nearly-exhaustive ground state searches. This Hamiltonian, commonly referred to as a cluster expansion (CE), can, with the ansatz that the atoms of the stable structures lie approximately on an fcc lattice, identify all stable phases. The CE is trained using DFT-determined formation enthalpy data. [34, 9, 43, 13, 24, 29] Because the CE Hamiltonian can be evaluated very rapidly, millions of structures can be computed in minutes. Large lists of candidate structures, sometimes running into the hundreds of millions, can be enumerated for a given lattice and their energies computed via the CE.
Normally, a search of all superstructures up to 16 atoms/cell is sufficient for a full ground state characterization of a system. (Alloy crystal structures that are derivative superstructures, with unit cells larger than 12 atoms/cell, are rare.) However, for the present case it becomes necessary to extend the search to include structures that match the criteria set forth by DF. In this case, the concentration (15:17) and symmetry (cubic) of the phase in question provide the search criteria, and augmenting our search space to include all cubic, 32 atom unit cells with 15:17 stoichiometry will be sufficient to investigate the stability of the DF phase.

Enumerating all superstructures up to 16 atoms/cell was performed with well-established crystal structure enumeration algorithms [11, 12. The algorithm for concentration and symmetry restricted enumeration was more recently developed 14] and subsequently used to enumerate the cubic unit cells at 15:17 stoichiometry needed to extend the search space.

The CE model was constructed from a subset of the data of Fig. 3, with the remaining data being used to validate the model. The fitting procedure was performed using a Bayesian implementation of compressive sensing (BCS) 29] with a reweighting scheme used to decrease the complexity of the Hamiltonian. Four hundred data points were used as training data and the rms error over the validation set was $\sim 3 \mathrm{meV} /$ atom. The resulting Hamiltoninan was then used to compute the formation enthalpies of all enumerated structures $(\sim 160,000$ structures with 1-16 atoms per cell and $\sim 380,000$ structures with stoichiometry 15:17 and a cubic unit cell).

The CE data (Fig. 4) is mostly consistent with the DFT data shown in Fig. 3. Most importantly, the stability of the $\mathrm{L}_{1}$ structure at $50 \% \mathrm{Pt}$ is confirmed. On the other hand, the CE-predicted ground states differ from the DFT ground states for Ag-rich compounds. This is not surprising given the fact that they are within the CE's margin of error $(\sim 3 \mathrm{meV} /$ atom $)$ of the convex hull for Ag-rich compounds. Such structures would be difficult to detect experimentally - they are so shallow on the convex hull that they would be kinetically inhibited at temperatures well above their order-disorder transition.

Most noteworthy is the absence of a breaking point on the convex hull at 15:17 stoichiometry, indicating that a phase separated mixture of $\mathrm{L}_{1}$ and pure $\mathrm{Pt}$ is favorable over an ordered compound 
here. Further investigation of the lowest energy 15:17 phase indicates a very $\mathrm{L} 1_{1}$-like arrangement of atoms (hereafter referred to as "the DF hypothetical phase" or ). The DF hypothetical phase is very similar to $\mathrm{L} 1_{1}$ with both phases exhibiting an AB stacking pattern in the [111] direction. The DF hypothetical phase differs from $\mathrm{L} 1_{1}$ by introducing $\mathrm{Pt}$ atoms in normally pure $\mathrm{Ag}$ planes of $\mathrm{L} 1_{1}$.

\subsection{Alternate Hypotheses}

Assuming that the composition reported in DF is correct (that there is, at finite temperatures at least, a stable structure at 53\%) and that the calculations are also correct, three possibilities remain: (1) the structure is stable only at finite temperatures $\left(\mathrm{L}_{1}\right.$ being stable as $\left.T \rightarrow 0 \mathrm{~K}\right),(2)$ the structure has a unit cell larger than 32-atoms (highly improbable but there are a few ordered structures with unit cells around 50 atoms [See some platinum group metal examples in Ref. [15]]), or (3) the structure is not fcc-based.

The testing of possibility (1) is discussed in the next section. To test possibility (2), we can use Monte Carlo simulations of the CE Hamiltonian. Using simulated annealing (SA), the stability of large unit cells can be checked. There is no guarantee that a structure below the tie-line between $\mathrm{L} 1_{1}$ AgPt and pure Pt will be found, even if it exists, but if the driving force for ordering is strong enough for the phase to be found in experiment, it would be likely that the structure would also be found in simulated annealing.

Possibility (3) is a very improbable case. Although ordered structures between two fcc elements sometimes adopt structures that are not derivatives of the underlying fcc lattice, they typically adopt relatively well-known structures. Such structures are already included in the AfLow libary 4, 2 and were checked in our initial DFT calculations (a superset of those we used to develop the CE). The only way for this case to be tenable is for the DF structure to be non-fcc-based and outside of the list of known cases. This is a very difficult case to rule out, despite its obvious unlikelihood. Nonetheless we undertook a computational search for such structures using the "symmetry-adapted" search of Meredig et al. 26]

For this search, we employed a very extensive crystal structure search (through hundreds of space groups and tens of thousands of candidate structures) using the symmetry-enforcing approach of first principles-assisted structure solution
(FPASS). 26 In FPASS, which may also be used to solve crystal structures from X-ray diffraction patterns, a genetic algorithm searches within a particular space group for low-energy structures by testing various occupations of Wyckoff positions and optimizing the values of internal parameters associated with those positions.

In the case of the 15:17 structure search, the correct space group was not known a priori; hence, we undertook an exhaustive search across possible space groups. As such a search would have been computationally prohibitive to perform with first-principles methods such as DFT, we first used a technique called iterative potential refinement (IPR) 39, 40 to construct an empirical embedded atom method (EAM) potential[19 for the AgPt system using a series of DFT structures, energies, and forces as training input. With this much more computationally efficient surrogate for DFT in place, we were able to feasibly scan through a large number of space groups and candidate structures.

The lowest-energy structure revealed by this high-throughput crystal structure search at 15:17 composition belongs to space group \#148, $R \overline{3}$, with EAM-relaxed lattice parameters of $a=9.06$ Aand $c=22.30 \AA$. Upon further examination, we identified this structure as a slightly distorted fcc supercell, that shown in Fig. 4. It is the same DF hypothetical structure as that identified by the cluster expansion modeling. Thus, while our FPASS-based structure search evaluated a vast, unconstrained configuration space of possible non-fcc lattices, it ultimately identified an fcc-based superstructure (the same found by the $\mathrm{CE}$ ) as the most favorable phase at the 15:17 stoichiometry.

At the same time, both the CE-based, "largecell" ground state search and the FPASS approach find no structure at or around $53 \%$ below the tie-line of $\mathrm{L}_{1}$ and pure $\mathrm{Pt}$, but both find the same lowest-energy (but unstable) structure at $53 \%$. This $\mathrm{L} 1_{1}$-like hypothetical structure is a 32 atom, cubic fcc-superstructure that strongly resembles $\mathrm{L} 1_{1}$ but has a stoichiometry of 15:17. The "extra" (relative to $\mathrm{L}_{1}$ ) Pt atoms form an ordered array at the corners of a 32-atom simple-cubic supercell, as shown in Fig. 4. If the $53 \%$ DF phase is truly stable at and above room temperature, then it is likely to be this structure (or to resemble it very closely) because this has the lowest formation enthalpy of any structure tested computationally. This structure will be re-examined in the following 


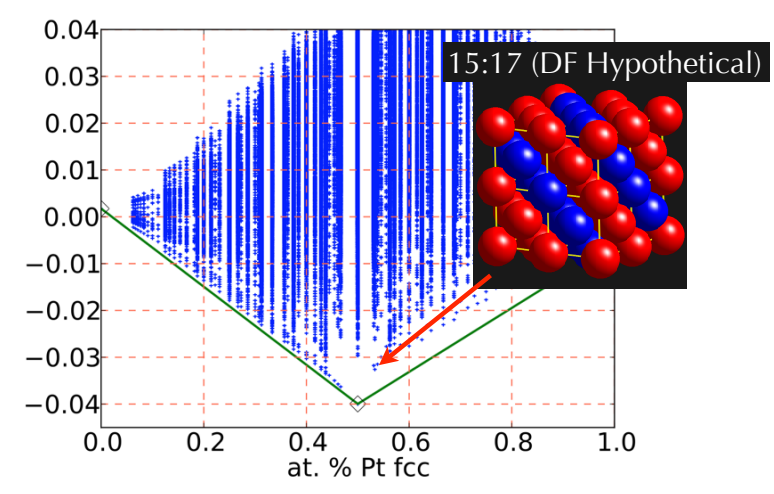

Figure 4: Formation enthalpies of candidate Ag-Pt compounds for $\approx 540,000$ structures calculated via cluster expansion. Included in this search are $\approx 380,00032$ atoms/cell structures with cubic symmetry and 15:17 stoichiometry. The convex hull construction indicates two stable phases at low temperatures: $\mathrm{L}_{1}$ and $50 \% \mathrm{Pt}$ and a 3 -atom/cell structure at $33 \%$ (an $\mathrm{A}_{2} \mathrm{~B}$ stacking of $\mathrm{Ag} / \mathrm{Pt}$ in the $\mathrm{XYZ}$ direction). Inset: the DF hypothetical structure, the lowest enthalpy structure at $15: 17$, is similar to $\mathrm{L} 1_{1}$. The only difference is that the atoms at the corners of the cell are $\mathrm{Pt}$ instead of $\mathrm{Ag}$ (red instead of blue).

section.

\section{Finite temperature phases}

As mentioned above, if the $53 \%$ DF hypothetical phase of DF is stable only at elevated temperatures, and the computationally-predicted phase of the $\mathrm{L} 1_{1}$ structure at $50 \%$ is only stable at low temperatures, the DF work and the present work may be complementary and not in conflict. A number of computational studies have indicated $\mathrm{L}_{1}$ as a ground state, but all have been predictions based on totalenergy calculations, without appeal to the entropic effects of finite temperature except the study by Sluiter et al. 38. In that study (Fig. 14(d) therein), only a narrow $\mathrm{AgPt}$ single phase region is seen up to about $1000 \mathrm{~K}$ and there is no hint of a stable compound near $\mathrm{Ag}_{15} \mathrm{Pt}_{17}$ at elevated temperature. Only configurational entropy effects are included in that study, but as results below suggest, vibrational contributions might not significantly affect the competition between $\mathrm{AgPt}$ and $\mathrm{Ag}_{15} \mathrm{Pt}_{17}$. Perhaps, the $\mathrm{L} 1_{1}$ phase at elevated temperatures has a smaller entropy (larger free energy) for configurational defects than the lowest-energy 32-atom, 15:17 DF hypothetical structure mentioned above. If so, it could be entropically stabilized at higher temperatures, explaining why a $53 \%$-Pt phase is seen in experiment.

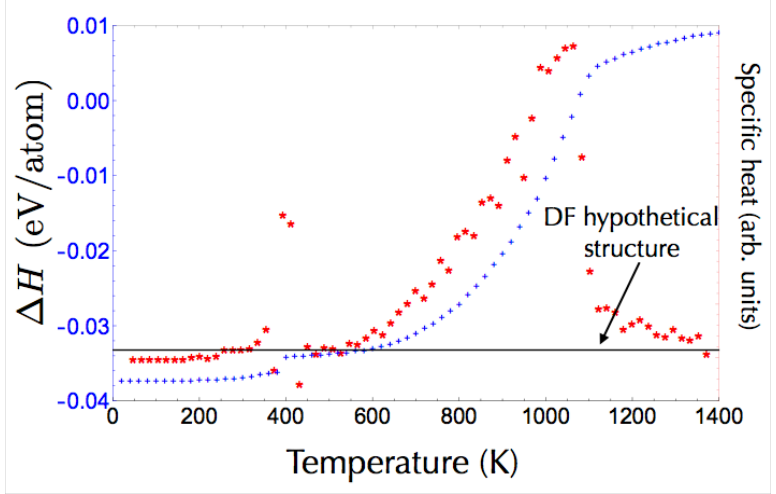

Figure 5: Chemical energy (blue) and heat capacity (red) vs. temperature for $\mathrm{a} \approx 16,000$ atom thermodynamic Monte Carlo simulation at 15:17 stoichiometry. The horizontal, dashed line is the energy of the reported $\mathrm{Ag}_{15} \mathrm{Pt}_{17}$ phase. The energy converged to a value that is lower in energy than the DF hypothetical phase suggesting the presence of a phase seperated mixture of $\mathrm{L}_{1}$ and $\mathrm{Pt}$.

To investigate the possibility that configurational entropy stabilizes $\mathrm{Ag}_{15} \mathrm{Pt}_{17}$, annealing of a 15:17 sample was simulated via thermodynamic Monte Carlo. The simulation cell contained 16,384 total atoms and was chosen to be cubic $(16 \times 16 \times 16)$ to ensure commensurability with the phase in question. Cell energies were averaged over $10^{7}$ spin flips, and the cell was considered converged when the difference between subsequent energy averages differed by less than $10^{-4} \mathrm{eV} /$ atom. Results from the simulation are given in Fig. 5, with chemical energy and heat capacity given as a function of temperature. If the phase in question were stable at finite temperature, a plateau in the energy would occur at or above the energy of the single-crystal DF hypothetical phase energy $(\Delta H=-0.03323 \mathrm{eV} /$ atom $)$. A plateau at the DF hypothetical phase energy is possible only if the cell converged to a perfect single crystal of the hypothetical phase. A plateau slightly above this energy could indicate the presence of grain boundaries separating domains of $\mathrm{Ag}_{15} \mathrm{Pt}_{17}$. Instead, we see a plateau forming slightly below the energy of the DF hypothetical phase, which indicates the presence of a phase separated mixture of $\mathrm{L}_{1}$ and $\mathrm{Pt}$. These results give no convincing evidence for an entropically-stabilized phase at 15:17 stoichiometry.

The MC simulations only test configurational entropy. Another, but usually smaller finitetemperature effect, is that of vibrational entropy. Although unlikely, the possibility that the DF hypo- 
thetical 32-atom structure is stabilized by phononic effects was also checked by first principles calculations. The computed phonon densities of states (PDOS) for fcc Ag, fcc Pt, L1 1 and the hypothetical phase are shown in Fig. 6.

The PDOSs of pure $\mathrm{Ag}$ and $\mathrm{Pt}$ are very similar in shape: typical Debye-like low frequency modes, a local maximum at a frequency of about half the upper frequency edge, and a maximum PDOS immediately below the upper frequency edge. The most significant difference in the PDOS of $\mathrm{Ag}$ and $\mathrm{Pt}$ is the frequency scale: the more rigid bonds between Pt atoms cause higher frequencies than occur in $\mathrm{Ag}$, in spite of the lower mass of $\mathrm{Ag}$ atoms. The PDOS of $\mathrm{L}_{1}$ resembles roughly the weighted sum of the Ag and Pt PDOS. The individual upper frequency edges of $\mathrm{Ag}$ and $\mathrm{Pt}$ can be clearly distinguished. There is a slight shift of the spectrum towards higher frequencies as is often seem for stable compounds, which have after all stronger interatomic bonds than the constituent pure elements.

The PDOS of the $\mathrm{L} 1_{1}$ and DF hypothetical structures $\left(\mathrm{Ag}_{15} \mathrm{Pt}_{17}\right)$ strongly resemble each other, although it can be remarked that the Ag-like upper frequency edge maximum is a little less pronounced and that the upper frequency edge goes to higher values for the Pt-rich DF hypothetical structure. The minor upward frequency shift in $\mathrm{L}_{1}$ relative to the constituent elements suggests that vibrational excitations are fewer in the $\mathrm{L}_{1}$ phase than in pure $\mathrm{Ag}$ and $\mathrm{Pt}$, so that vibrational effects can be expected to disfavor compound formation. This is born out in the vibrational contribution to the free energy of compound formation

$\Delta F_{\mathrm{vib}}\left[\operatorname{Ag}_{n} \mathrm{Pt}_{m}\right]=F_{\mathrm{vib}}\left[\mathrm{Ag}_{n} \mathrm{Pt}_{m}\right]-n F_{\mathrm{vib}}[\mathrm{Ag}]-m F_{\mathrm{vib}}[\mathrm{Pt}]$,

where $F_{\text {vib }}$ is computed from the PDOS (see, e.g., Ref. [37]). Neither $\mathrm{L} 1_{1}$ or the DF hypothetical is favored as is apparent from Fig. 7, although the magnitude of $\Delta F_{\text {vib }}$ is rather small, even at $1000 \mathrm{~K}$ it still only compensates about half the $0 \mathrm{~K}$ formation enthalpy $38 . \mathrm{L} 1_{1}$ and the DF hypothetical exhibit very similar $\Delta F_{\text {vib }}$ values, so that vibrational effects cannot drive a preference for either structure. So neither configuration entropy nor vibrational entropy could account for high-temperature stability of the DF hypothetical $\left(\mathrm{Ag}_{15} \mathrm{Pt}_{17}\right)$ phase over the $\mathrm{L}_{1}$ AgPt phase, ruling out possibility (1) (introduced at the beginning of Sec. 2.3.

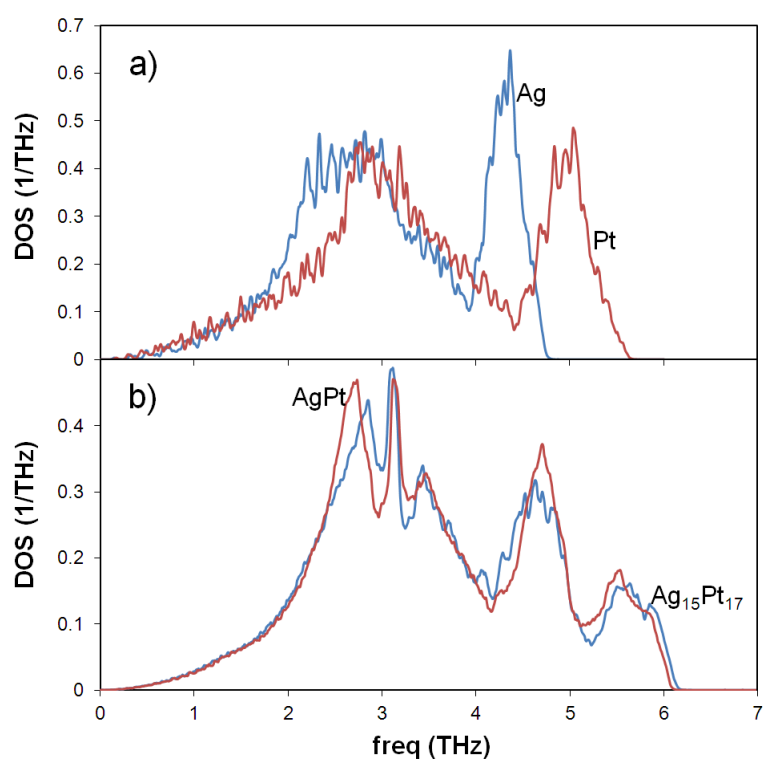

Figure 6: Normalized phonon density of states: a) fcc Ag (blue) and fcc Pt (red); b) $\mathrm{Ag}_{15} \mathrm{Pt}_{17}$ (blue) and $\mathrm{L}_{1}$ type AgPt (red).

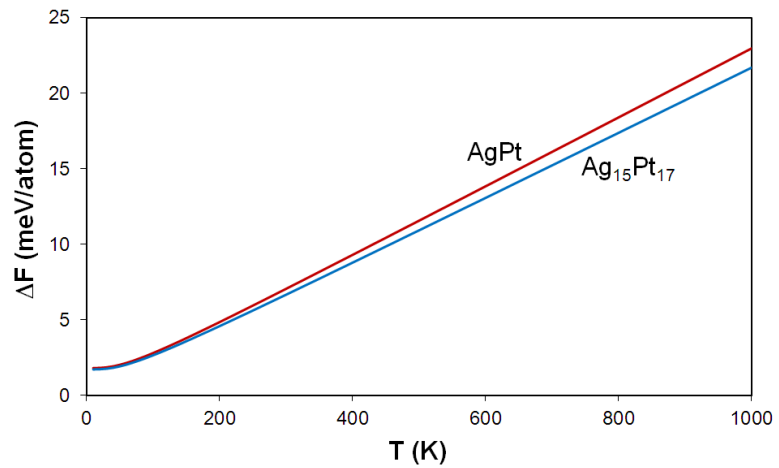

Figure 7: Vibrational contribution to the free energy of compound formation with reference to fcc $\mathrm{Ag}$ and fcc Pt as function of temperature $T$, see eqn. $1 . \mathrm{Ag}_{15} \mathrm{Pt}_{17}$ (blue) and $\mathrm{L} 1_{1}$ type $\mathrm{AgPt}$ (red). 


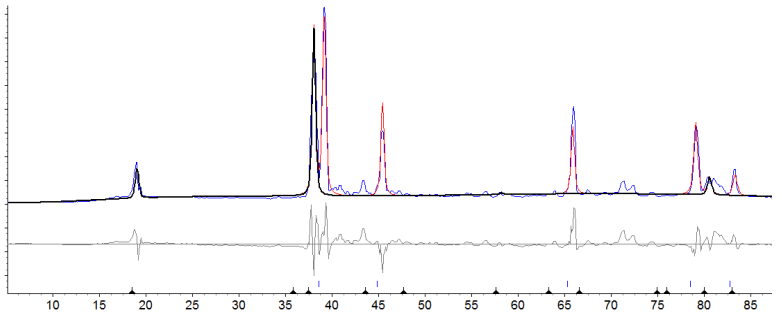

Figure 8: Rietveld fit of a two phase model against an xray powder diffraction dataset extracted from the scanned image of Figure 4 from Ref. 7]. The model includes a fullydisordered PtAg FCC model, together with a small $\mathrm{L1}_{1}$ ordered component based on a slightly-larger underlying cell parameter and severe preferred orientation (see text for details and qualifications). Red indicates the observed pattern, blue indicates the calculated pattern, gray indicates the difference (obs-calc) pattern. The heavy black line indicates the contribution of the $\mathrm{L} 1_{1}$-ordered phase to the calculated pattern. The upper blue tick marks and lower black tick marks with triangles below the pattern indicate the Braggpeak positions associated with the majority and minority phases, respectively. The horizontal scale is $2 \theta$ in degrees.

\section{Re-examination of Durussel-Feschotte X- ray Diffraction Data}

To more carefully examine the data and structural claims of DF, we digitally extracted a lowquality dataset from a scanned copy of their Fig. 4 (details in the appendix.) The extracted diffraction dataset was subjected to Rietveld analysis using the TOPAS-Academic v5 software. See the resulting fit in Fig. 8,

It was straightforward to fit the cubic parent peaks to a fully-disordered fcc model using a single cubic cell parameter of $a=4.038 \AA$, roughly halfway between the values expected for Pt (3.9239 $\AA)$ and $\mathrm{Ag}(4.0853 \AA)$. We checked the diffraction pattern from contributions from a variety of potentially-relevant oxides of $\mathrm{Pt}$ and $\mathrm{Ag}$, but found no evidence of an oxide impurity. Because the spurious peak near 38 degrees is so large and so close to the fcc-parent 111 peak, we investigated the possibility of a second cubic fcc phase. But such a model fails to contribute at nearly all of the expected higher-angle peak positions. However, by assuming nearly-perfect preferred orientation of the 111 fcc axis of the second phase normal to the sample plane, it was possible to fit the 38-degree peak fairly well using a cell parameter of $a_{s}=4.152 \AA$. We then found that adding $\mathrm{L}_{1}$ order to the rhombohedral supercell associated with this slightly larger fcc cell $\left(a_{r}=a_{s} / \sqrt{2}\right.$ and $c_{r}=a_{s} 2 \sqrt{3}$ nicely reproduces the position and relative intensity of the substantial su- perlattice peak near 19 degrees, which indexes as 003 in a hexagonal setting. So while an $\mathrm{L}_{1}$ ordering would account for most prominent superlattice peak, it would not account for many of the smaller spurious peaks at higher angles.

The refined mass fraction of the $\mathrm{L} 1_{1}$ phase was only $1.3 \%$. But like the peak at 38 degrees, which indexes to 006 for the ordered supercell, the large relative intensity of the 003 peak would presumably be due to extreme preferred orientation. Yet, no preferred orientation of the cubic phase was evident. One could envision a flat sample consisting of randomly-oriented grains of the disordered cubic parent phase, upon which a film of the $\mathrm{L} 1_{1}$-ordered phase grows, with the hexagonal 001 axis always normal to the flat surface. Because this seems unlikely; we instead conclude that the DF data in Ref. [7] is problematic, and that a comparable experiment should be repeated. In summary, whereas DF use the position of the 19-degree peak to imply an Ag:Pt/15:17 ordering, we demonstrate that the same peak is also consistent with the much simpler $L 1_{1}$ ordering (predicted by several computational studies, including ours). Our experimental results, reported below, examine multiple new samples and take a much more careful approach to structural analysis (TEM) and composition measurement (microprobe) than that reported in the DF work and are entirely consistent with the simpler interpretation of a stable $\mathrm{L} 1_{1}$ phase at 50:50.

\section{Experimental results}

We studied two compositions experimentally (see Sec. 8.4 for sample preparation discussion), 51 at. $\%$ and 54 at.\% platinum. Images were acquired by SEM, using BSE to show differences in atomic number (the heavier element, $\mathrm{Pt}$, shows brighter contrast than the lighter element, $\mathrm{Ag}$ ). After heat treatment at $850^{\circ} \mathrm{C}$ for 40 days, both experimental alloys showed a two-phase microstructure. The composition of the phases was (Ag-91.5 at.\%Pt, Ag36.5 at.\%Pt) and (Ag-92.4 at.\%Pt, Ag-36.6 at.\%Pt) for the two alloys of overall composition 51 at. $\% \mathrm{Pt}$ and 54 at.\% $\mathrm{Pt}$, respectively. This phase separation into one platinum-rich and one silver-rich solid solution for samples with overall composition close to 50 at. $\% \mathrm{Pt}$ is consistent with the DF phase diagram (and other $\mathrm{Ag}$-Pt phase diagrams). In this phase diagram, there is a misibility gap above $803{ }^{\circ} \mathrm{C}$. Below this temperature there is a line phase, so we 
selected a lower temperature, $750^{\circ} \mathrm{C}$, to investigate the structure of the low-temperature phase.

Heat treatments at $750^{\circ} \mathrm{C}$ were carried out for lengthy periods in an attempt to achieve thermodynamic equilibrium and assure that the line phase appeared. Times of 20, 40 and 60 days showed progressive changes in the fraction of phases; there was however little change between 60 days and 80 days. (The results presented here for the $750{ }^{\circ} \mathrm{C}$ phase structure are for the sample annealed for 80 days.) The alloy sample of composition $\mathrm{Ag} 51.1$ at. $\% \mathrm{Pt}$ contained only a small fraction of the phases identified at $850{ }^{\circ} \mathrm{C}, 92.4$ at.\%Pt and 34.2 at.\%Pt, showing the appearance of a predominant third phase. The third phase had a composition of $\mathrm{Ag} 48$ at.\%:Pt 52 at.\%, as determined by EDS. The alloy sample of composition $\mathrm{Ag} 46$ at.\%:Pt 54 at.\% also showed a third phase of composition Ag 48 at.\%:Pt 52 at.\%. Like the EPMA data of DF, EDS is subject to large error bars. These measured compositions do not give preference either to the DF 53 at.\%-Pt phase or to the 50:50 $\mathrm{L}_{1}$ phase.

Hardness measurements, conducted on both alloys after each heat treatment, showed an increase in hardness (relative to both the as-cast and asrolled alloys) after heat treatment at $750^{\circ} \mathrm{C}$. This change in hardness increased with length of heat treatment so that the greatest increase was observed after heat treatment for 80 days at $750^{\circ} \mathrm{C}$. Ordered structures impede dislocation motion and thus exhibit higher hardness than their disordered counterparts, so an increase in hardness with heat treatment time is consistent with the growth of an ordered phase The observed hardness increases with increasing annealing times (20, 40, 60, 80 days) are therefore consistent with the growth of a third phase, the volume fraction of the third phase increasing with annealing time. Subsequent heat treatment above the transformation temperature of $803^{\circ} \mathrm{C}$, at $850^{\circ} \mathrm{C}$, resulted in a decrease in hardness consistent with the dissolution of the third phase: the hardness reverts to that of the as-cast alloy.

The hardness measurements are consistent with the presence of a third, ordered phase (composition near 50:50) which develops below temperatures of $800 \mathrm{C}$. The measurements do not answer the question whether this third phase is $\mathrm{L}_{1}$ or a large-unit-cell cubic phase as conjectured by DF. High-resolution HAADF STEM analysis shows that the samples contain small $(\sim 10 \mathrm{~nm})$ domains of fcc solid solution and ordering with $\mathrm{Ag}$ and $\mathrm{Pt}$ atoms arranged on $\{111\}$ planes. See Fig. 9. The ordering
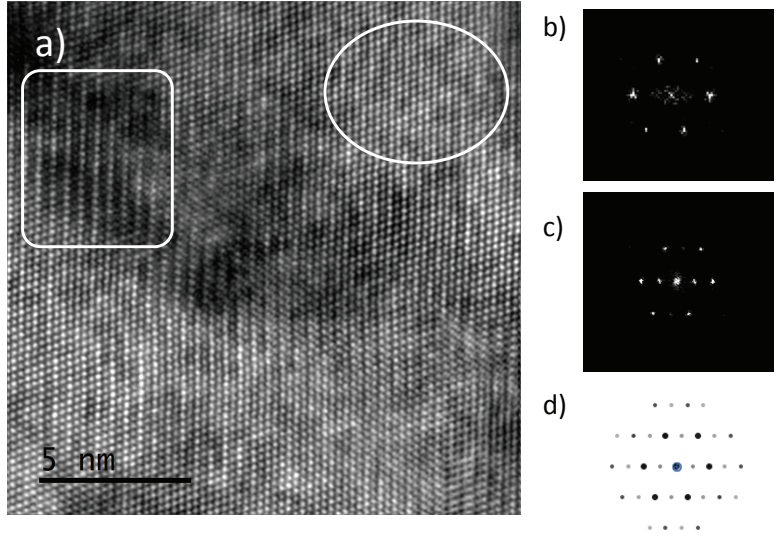

Figure 9: Panel (a): HAADF STEM micrograph of the Ag$\mathrm{Pt}$ alloy with the beam along the [110] direction of the $\mathrm{L} 1_{1}$ phase. The brighter rows of the atoms (rectangular region) are platinum planes, dimmer rows silver planes. The region in the ellipse is a region where the platinum and silver atoms are disordered. Panel b): FFT of the disordered region (ellipse) of a). Panel c): FFT of the ordered region (rectangle) in panel a). Panel d): Calculated diffraction pattern of the $\mathrm{L} 1_{1}$ phase with the beam along the [110] direction.

along 111 planes is consistent with both structures. Both structures would lead to the appearance of superlattice reflections (as in panel c)). If this third phase was the DF hypothetical phase instead of $\mathrm{L} 1_{1}$ there would be a second set of superlattice reflections due to the in-plane ordering of defects in the $\{111\}$ planes of silver. However, the intensity of these peaks is predicted to be only $10^{-5}$ that of the primary fcc peaks. Even if they were present, they would be difficult to observe. Thus, the STEM results do not rule out either the $\mathrm{L} 1_{1}$ phase or the DF hypothetical phase. Hardness measurements and SEM/BSE clearly show the existence of some third phase with a concentration near 50 at.\%-Pt, and the STEM results give strong evidence for an ordering of $\{111\}$ planes, a feature of both the DF hypothetical phase and $\mathrm{L} 1_{1}$.

Because of their structural similarity, the best way to discriminate between the two phases would be a stringent test of their compositions. We performed additional microprobe analysis on our samples. Standards consisted of presumably pure samples of $\mathrm{Ag}$ and $\mathrm{Pt}$ taken from sputtering targets. Standards and samples of interest were freshly polished before analysis and EDS results showed no measureable constituents in standards or sample other than the expected $\mathrm{Ag}$ and Pt. Figure 10 shows an image of a nominally 53/47 AgPt alloy sample that was annealed for 60 days at $750 \mathrm{C}$. 


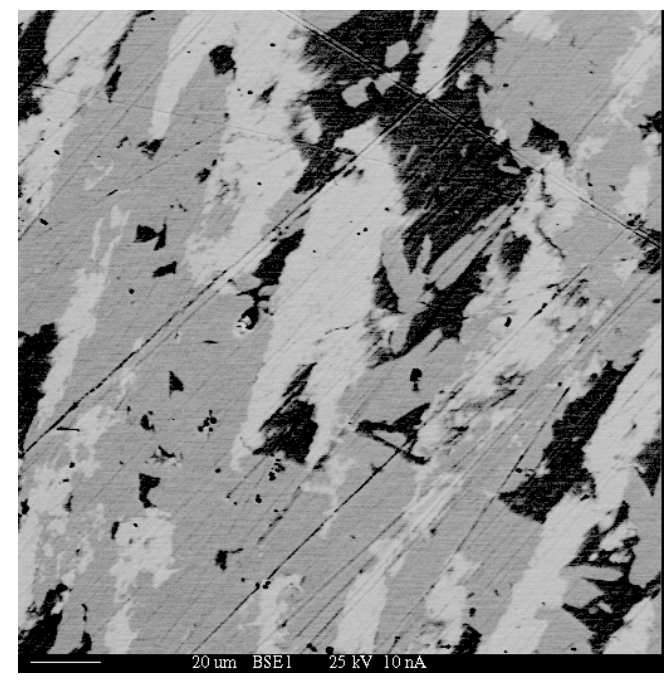

Figure 10: SEM (Cameca SX-50 microprobe) backscattered electron image of a nominally 53/47 Ag-Pt alloy that was annealed at $750^{\circ} \mathrm{C}$ for 60 days. Dark, light, and gray phases are visible with compositional data showing that color reflects the relative $\mathrm{Pt} / \mathrm{Ag}$ composition. The gray phase is near 50:50 and the focus of this manuscript.
Dark, light, and gray phases in the image reflect the relative $\mathrm{Pt} / \mathrm{Ag}$ concentration. Composition analysis was done in a Cameca SX-50 microprobe at $25 \mathrm{kV}$ and $10 \mathrm{nA}$ using the $\mathrm{Ag}$ and $\mathrm{Pt} \mathrm{L}$ edges and the Cameca software quantification and correction routines. Three different gray grains and a total of 13 spots were analyzed. All pre-normalization concentrations were within $1 \%$ of $100 \%$ with the average being $99.60 \%$. The normalized $\mathrm{Pt}$ concentration from these spots was $50.68 \% \pm 0.20 \%$. The error of $0.20 \%$ is a statistical spread within the data set analyzed and does not reflect any potential systematic errors associated with the experimental setup or correction approaches. Our analysis includes prenormalization data and data about standards and experimental conditions, and thus to us seems more reliable than the DF results and more indicative of the 50:50 L1 1 phase. However, both our and the DF analysis are plagued by the significant spread in the correction factors for $\mathrm{Ag} \mathrm{L}$. With no clear guidelines of which correction approach is better, the experimental data is unable to definitively distinguish between the DF hypothetical structure and $\mathrm{L} 1_{1}$.

\section{Summary}

The general features of the DF phase diagram[7] are correct: A single ordered structure around 50 at.\% in a large miscibility gap, with a transition temperature $\sim 1000 \mathrm{~K}$. The notable absence of compounds at other concentrations in the DF phase diagram, in contrast to earlier phase diagrams, is consistent with our computational predictions. However, the ordered compound in the middle of the miscibility gap has a $\mathrm{Pt}$ concentration of $50 \%$ (rather than 53\%). Its structure is the simple $\mathrm{L} 1_{1}$ structure that one would expect, in analogy to $\mathrm{Cu}$ $\mathrm{Pt}$, and consistent with several DFT-based computational predictions and extensive calculations herein.

Furthermore, our calculations indicate the possibility of Ag-rich ordered phases in the solid-solution field of the DF diagram. As expected, the nature of the ordering for these phases is similar to $\mathrm{L} 1_{1}$, with stacking patterns in the 111 direction at 3:8 and 3:7 stoichiometry. However, due to the shallow depth of the convex hull at these concentrations, it is likely that these orderings happen either below the temperatures reported in the earlier phase diagram or, more likely, that they happen slowly 
due to sluggish kinetics, making them difficult to synthesize.

Another notable aspect of this work is support it lends to high-throughput studies. [5] In HT studies, computation often finds more ground states than reported in experiment. Occasionally, there are direct contradictions, especially when the reported experimental structures are speculative. This thorough re-examination of the Ag-Pt phase diagram, comparing computation and experiment, gives strong support for the HT mode of discovery and is important for MGI goals, validating HT searches using DFT (and cluster expansion). Finally, as a point of curiosity, $\mathrm{L} 1_{1}$ has never been seen experimentally outside of the $\mathrm{Cu}-\mathrm{Pt}$ system. Computational predictions of other systems with the $\mathrm{L}_{1}$ structure are reported in Ref. [28].

\section{Acknowledgments}

G. L. W. H. and L. J. N. are grateful for financial support from the NSF, DMR-0908753. J. H. N. and E. J. O. acknowledge the financial support from the NRF and DST. We appreciate helpful discussions about microprobe analysis with John Fournelle and Mike Dorais. B. M. (FPASS calculations) acknowledges support by the U.S. Department of Energy, Office of Science, Basic Energy Sciences, under Grant No. DEFG02-07ER46433. C. W. acknowledges financial assistance award 70NANB14H012 from U.S. Department of Commerce, National Institute of Standards and Technology as part of the Center for Hierarchical Materials Design (CHi$\mathrm{MaD})$.

\section{Methods}

\subsection{Phonon calculations}

The so-called direct method was used for extracting $3 \times 3$ force constant matrices [36]. In 64-atom cells, with fcc translations $<220>$, one atom at a time was displaced in $\mathrm{x}, \mathrm{y}$, or $\mathrm{z}$ direction by about $0.003 \mathrm{~nm}$ and resulting Hellmann-Feynman forces on all other atoms within the 64 atom cell were then computed using VASP (version 4.6.36). Force constants up to, and including, the seventh neighbor shell $\left(<\frac{3}{2} 1 \frac{1}{2}>\right)$ were determined for fcc Ag, fcc Pt, $\mathrm{L}_{1} \mathrm{AgPt}$, and $\mathrm{Ag}_{15} \mathrm{Pt}_{17}$ structures while imposing the translational, rotational, and other invariances [36]. The largest element in the $3 \times 3$ force constant matrices was at least 3 orders of magnitude smaller for the seventh shell than for the first shell, indicating that the force constants rapidly decayed as interatomic distance increased.

Crystal structures of fcc $\mathrm{Ag}$ and $\mathrm{Pt}$, and of $\mathrm{L}_{1}$ $\mathrm{AgPt}$ and $\mathrm{Ag}_{15} \mathrm{Pt}_{17}$ were relaxed such that stresses were converged to within $1 \mathrm{kBar}$ and atomic forces had magnitudes below $1 \mathrm{meV} /$ Angstrom. Lattice parameters of underlying fcc structure were 0.41594 (0.39717) $\mathrm{nm}$ for $\mathrm{Ag}(\mathrm{Pt})$. The AgPt and $\mathrm{Ag}_{15} \mathrm{Pt}_{17}$ featured a small extension along the [111] direction normal to the $\mathrm{L} 1_{1}$ composition modulation. Therefore, the interatomic distances in the [111] direction was $3.32 \%$ (4.26\%) greater than in the (111) planes for $\mathrm{AgPt}\left(\mathrm{Ag}_{15} \mathrm{Pt}_{17}\right)$. The fcc lattice parameters based on the cube root of the volume per 4 atoms was 0.40395 (0.40378) nm for $\mathrm{AgPt}\left(\mathrm{Ag}_{15} \mathrm{Pt}_{17}\right)$ corresponding to a lattice parameter contraction of less than $1 \%$ relative to the average of the pure fcc elements. The phonon density of states (PDOS) was obtained by diagonalizing the dynamical matrix for 8000 homogeneously distributed k-points in the first Brillouin zone of the 64 atom cell and broadening the resulting spectrum by $0.1 \mathrm{meV}$.

\subsection{DFT calculations}

The chemical energies of all crystal structures were calculated from the density-functional theory (DFT) using the VASP software. [23, 22] We used projector-augmented-wave (PAW) potentials [1] and the generalized gradient approximation (GGA) to the exchange-correlation functional proposed by Perdew, Burke and Ernzerhof. 32. To reduce random numerical errors, equivalent $k$-point meshes were used for Brillioun zone integration. 10] Optimal choices of the unit cells, using a Minkowski reduction algorithm, were adopted to accelerate the convergence of the calculations. 30 The effect of spin-orbit coupling was not included in our calculations because it's effect was shown to be a simple tilt of the calculated energies, as explained in Ref. [28] (see third paragraph in section II).

\subsection{X-ray analysis of DF data}

To more carefully examine the data and structural claims of [citation], we digitally extracted a low-quality dataset from a scanned copy of their Fig. 4. Using a photo-editor, the image was slightly derotated, converted from grayscale to black-white color scheme, and scrubbed of non-data pixels (e.g. axes, labels, legend, peak markers, and scan dirt). 
Then, a simple image-analysis code extracted the vertical pixel-height of the diffraction trace at each horizontal pixel position, averaging when the trace was multiple pixels thick, and treated this value as an effective diffraction intensity. The horizontal pixel positions were then matched up with the horizontal 2-theta scale from the original image. The extracted diffraction dataset was subjected to Rietveld analysis using the TOPAS-Academic v5 software. The fit is shown in Fig. 8 .

\subsection{Experimental methods}

Two alloy compositions were studied experimentally. These were made by melting platinum and silver granules, weighed out separately before melting. The overall compositions were determined by SEM/EDS to be Ag $51 \pm 1 \% \mathrm{Pt}$ and $\mathrm{Ag} 54 \pm 1 \% \mathrm{Pt}$ respectively. It should be noted that the as-cast alloys were not single-phase/homogeneous; the respective compositions are averages determined by acquiring several EDS spectra, from several wide areas, of several specimens.

The as-cast buttons were rolled to $90 \%$ reduction in thickness. Specimens were cut for heat treatment, SEM and hardness measurements from the rolled strips. Self-supporting discs, $3 \mathrm{~mm}$ in diameter with a thickness of 350-420 $\mu \mathrm{m}$, were punched from the rolled strips for heat treatment, DSC and TEM analysis.

Long heat treatments were carried out at elevated temperatures, terminated by quenching into water. Before heat treatments were conducted, specimens were coated with a ceramic paste to prevent oxidation. After heat treatment, coating removal was followed by mechanical grinding and polishing of the specimen surfaces, to remove any possible contamination.

HRSTEM specimens were prepared by using a Helios Nanolab 650 FIB SEM and investigated in a double Cs-corrected JEOL JEM-ARM200F operated at $200 \mathrm{kV}$.

\section{Microprobe compositions}

The DF model hinges on the microprobe derived composition of $53 \%$. However, that result has limited documentation of the data. Current standards in presenting microprobe results include presentation of analytical totals prior to normalization and details of the standards used. The DF Ag-Pt work makes reference to their paper on $\mathrm{PbPd}$ where comments are made to internal standards and frequent restandardization. Thus, correct use of standards is implied, but the specific details of the use of standards are lacking in the Ag-Pt paper. The composition data presented appear to be post normalization; this is where the measured compositions are renormalized to enforce a total composition of 100 percent. While this is a common approach, the presentation of prenormalization data allows the reader to understand the potential for systematic errors in the analysis. That is, confidence in the results is related to the degree that that the prenormalization concentrations add up to near 100 percent. The MAGIC IV algorithm was use by DF to implement matrix (ZAF) corrections. This algorithm is one of many that have been used over the years, each having different approaches and different tables of correction factors. In the case of $\mathrm{Ag} \mathrm{L}$ edges, the various $\mathrm{ZAF}$ routines have about a $15 \%$ variation (as per CALCZAF) while for $\mathrm{Pt}$ $\mathrm{L}$ it is near $1 \%$. This variation between correction approaches could result in a $3-4 \%$ systematic error in 50:50 Ag-Pt. While the DF approach of using pure element standards is widely practiced, however, there is evidence that it is inadequate when the mixture consists of significantly different atomic numbers 18. It is unclear if $\mathrm{Ag}$ and $\mathrm{Pt}$ are sufficiently different to exhibit this problem.

\section{References}

[1] P. E. Blöchl. Projector augmented-wave method. Phys. Rev. B, 50(24):17953, 1994.

[2] C. E. Calderon, J. J. Plata, C. Toher, C. Oses, O. Levy, M. Fornari, A. Natan, M. J. Mehl, G. Hart, M. B. Nardelli, et al. The aflow standard for high-throughput materials science calculations. Computational Materials Science, 108:233-238, 2015.

[3] S. Curtarolo, D. Morgan, and G. Ceder. Accuracy of ab initio methods in predicting the crystal structures of metals: review of 80 binary alloys. Calphad, 29:163, 2005.

[4] S. Curtarolo, W. Setyawan, G. L. W. Hart, M. Jahnatek, R. V. Chepulskii, R. H. Taylor, S. Wang, J. Xue, K. Yang, O. Levy, M. Mehl, H. T. Stokes, D. O. Demchenko, and D. Morgan. Aflow: an automatic framework for high-throughput materials discovery. Comp. Mat. Sci., 58:218-226, 2012. doi: 10.1016/j.commatsci. 2012.02.005.

[5] S. Curtarolo, G. L. W. Hart, M. B. Nardelli, N. Mingo, S. Sanvito, and O. Levy. The high-throughput highway to computational materials design. Nature Materials, 12(3):191-201, MAR 2013. ISSN 1476-1122. doi: 10. 1038/NMAT3568.

[6] F. Doerinckel. Metallographische mitteilungen aus dem institut für anorganische chemie der universität göttingen über einige platinlegierungen. Zeitschrift fr anorganische Chemie, 54:333-366, 1907. 
[7] P. Durussel and P. Feschotte. A revision of the binary Ag-Pt. Journal of Alloys and Compounds, 239:226-230, 1996.

[8] R. Erni, T. Etter, H. Heinrich, and G. Kostorz. On the formation of a silver-rich ordered phase in Ag-Pt solid solutions. ZEITSCHRIFT FUR METALLKUNDE, 92 (11):1194-1196, NOV 2001. ISSN 0044-3093.

[9] D. D. Fontaine. Cluster approach to order-disorder transformations in alloys, volume 47. Academic Press, New York, 1994.

[10] S. Froyen. Brillouin-zone integration by fourier quadrature: Special points for superlattice and supercell calculation. Phys. Rev. B, 3168, 1989.

[11] G. L. W. Hart and R. W. Forcade. Generating derivative structures: Algorithm and applications. Phys. Rev. B, 77:224115, 2008.

[12] G. L. W. Hart and R. W. Forcade. Generating derivative structures from multilattices: Application to hcp alloys. Phys. Rev. B, 80:014120, July 2009.

[13] G. L. W. Hart, V. Blum, M. Walorski, and A. Zunger Evolutionary approach for determination of firstprinciples hamiltonians. Nature Materials, 4:391-394, 2005.

[14] G. L. W. Hart, L. J. Nelson, and R. W. Forcade. Generating derivative structures for a fixed concentration. Comp. Mat. Sci., 59:101-107, 2012. doi: 10.1016/j.commatsci.2012.02.015

[15] G. L. W. Hart, S. Curtarolo, T. B. Massalski, and O. Levy. Comprehensive search for new phases and compounds in binary alloy systems based on platinumgroup metals, using a computational first-principles approach. Phys. Rev. X, 3:041035, Dec 2013. doi: 10.1103/PhysRevX.3.041035. URL http://link.aps . org/doi/10.1103/PhysRevX.3.041035

[16] G. L. W. Hart, S. Curtarolo, T. B. Massalski, and O. Levy. Comprehensive search for new phases and compounds in binary alloy systems based on platinumgroup metals, using a computational first-principles approach. Phys. Rev. X, 3:041035, Dec 2013. doi: 10.1103/PhysRevX.3.041035. URL http://link.aps. org/doi/10.1103/PhysRevX.3.041035.

[17] A. Jain, G. Hautier, C. J. Moore, S. P. Ong, C. C. Fischer, T. Mueller, K. A. Persson, and G. Ceder. A highthroughput infrastructure for density functional theory calculations. Comp. Mat. Sci., 50(8):2295-2310, 2011. doi: 10.1016/j.commatsci.2011.02.023.

[18] Y. A. C. John Fournelle, Chuan Zhang. Al-ir compounds and the problem of light element-heavy element matrix corrections in epma: Application of penepma monte carlo modeling. Microscopy and Microanalysis, 14, 2008. doi: 10.1017/S1431927608083992.

[19] R. Johnson. Alloy models with the embedded-atom method. Physical Review B, 39(17):12554, 1989.

[20] I. Karakaya and W. Thompson. The Ag-Pt (silverplatinum) system. Bulletin of Alloy Phase Diagrams, 8(4):334-340, 1987. ISSN 0197-0216. doi: 10. 1007/BF02869269. URL http://dx.doi.org/10.1007/ BF02869269

[21] W. Klement and H. Luo. Metastable solid solutions in silver-platinum alloys, 1963.

[22] G. Kresse and J. Furthmüller. Efficient iterative schemes for ab initio total-energy calculations using a plane-wave basis set. Physical Review B, 54(11169), 1996.

[23] G. Kresse and D. Joubert. From ultrasoft pseudopoten- tials to the projector augmented-wave method. Physical Review B, 59(1758), 1999.

[24] D. Lerch, O. Wieckhorst, G. L. W. Hart, R. W. Forcade, and S. Müller. Constructing cluster expansions for arbitrary lattices from minimal user input. Model. Simul. Mater. Sci. Eng., 17:055003, May 2009.

[25] T. B. Massalski, H. Okamoto, P. R. Subramanian, and L. Kacprzak, editors. Binary Alloy Phase Diagrams. American Society for Metals, Materials Park, OH, 1990.

[26] B. Meredig and C. Wolverton. A hybrid computationalexperimental approach for automated crystal structure solution. Nature materials, 12(2):123-127, 2013.

[27] S. Müller and A. Zunger. First-principles predictions of yet-unobserved ordered structures in the $\mathrm{Ag}-\mathrm{Pd}$ phase diagram. Phys. Rev. Lett., 87(16):165502, Sep 2001.

[28] L. J. Nelson, G. L. W. Hart, and S. Curtarolo. Groundstate characterizations of systems predicted to exhibit $l 1_{1}$ or $l 1_{3}$ crystal structures. Phys. Rev. B, 85:054203, Feb 2012. doi: 10.1103/PhysRevB.85.054203.

[29] L. J. Nelson, V. Ozolins, C. S. Reese, F. Zhou, and G. L. W. Hart. Cluster expansion made easy with Bayesian compressive sensing. Physical Review B, 88 (15), OCT 3 2013. ISSN 1098-0121. doi: 10.1103/ PhysRevB.88.155105.

[30] P. Q. Nguyen and D. Stehlé. Low-dimensional lattice basis reduction revisited. ACM Trans. Algorithms, 5 (4):1-48, Nov. 2009. ISSN 1549-6325. doi: 10.1145/ 1597036.1597050. URL http://doi.acm.org/10.1145/ 1597036.1597050

[31] H. Okamoto. Ag-Pt (silver-platinum). Journal of Phase Equilibria, 18(5):485-485, 1997. ISSN 1054-9714. doi: 10.1007/BF02647706. URL http://dx.doi.org/ 10.1007/BF02647706

[32] J. P. Perdew, K. Burke, and Ernzerhof. Generalized gradient approximation made simple. Phys. Rev. Lett., 77:3865, Oct. 1996.

[33] A. V. Ruban, S. I. Simak, P. A. Korzhavyi, and B. Johansson. Theoretical investigation of bulk ordering and surface segregation in Ag-Pd and other isoelectornic alloys. Physical Review B, 75(5), FEB 2007. ISSN 10980121. doi: $\{10.1103 /$ PhysRevB.75.054113\}.

[34] J. M. Sanchez, F. Ducastelle, and D. Gratias. Generalized cluster description of multicomponent systems. Physica A, 128:334-350, 1984.

[35] A. Schneider and U. Esch. Das system silber-platin. Z. Elecktrochem., 49, 1943.

[36] M. H. F. Sluiter, M. Weinert, and Y. Kawazoe. Determination of the elastic tensor in low-symmetry structures. EPL (Europhysics Letters), 43(2):183, 1998. URL http://stacks.iop.org/0295-5075/43/i=2/a=183

[37] M. H. F. Sluiter, M. Weinert, and Y. Kawazoe. Force constants for substitutional alloys. Phys. Rev. B, 59:4100-4111, Feb 1999. doi: 10.1103/PhysRevB. 59.4100. URL http://link.aps.org/doi/10.1103/ PhysRevB.59.4100

[38] M. H. F. Sluiter, C. Colinet, and A. Pasturel. Ab initio calculation of the phase stability in Au-Pd and Ag-Pt alloys. Phys. Rev. B, 73(17):174204, May 2006. doi: 10.1103/PhysRevB.73.174204.

[39] A. E. Thompson, B. Meredig, M. Stan, and C. Wolverton. Interatomic potential for accurate phonons and defects in uoj subi, $2 \mathrm{i} /$ subi. Journal of Nuclear Materials, 446(1):155-162, 2014.

[40] A. E. Thompson, B. Meredig, and C. Wolverton. An improved interatomic potential for xenon in uo2: a com- 
bined density functional theory/genetic algorithm approach. Journal of Physics: Condensed Matter, 26(10): 105501, 2014.

[41] P. Villars, H. Okamoto, and K. Cenzual, editors. ASM Alloy Phase Diagrams Center. http://www1.asminternational.org/AsmEnterprise/APD, ASM International, 2006-2014.

[42] K. Yun, Y.-H. Cho, P.-R. Cha, J. Lee, H.-S. Nam, J. S. Oh, J.-H. Choi, and S.-C. Lee. Monte carlo simulations of the structure of pt-based bimetallic nanoparticles. Acta Materialia, 60(12):4908-4916, JUL 2012. ISSN 1359-6454. doi: 10.1016/j.actamat.2012.05.032.

[43] A. Zunger. First-principles statistical mechanics of semiconductor alloys and intermetallic compounds. In P. E. A. Turchi and A. Gonis, editors, Statics and Dynamics of Alloy Phase Transitions, NATO ASI Series, Ser. B, pages 361-419, New York, 1994. Plenum Press. 


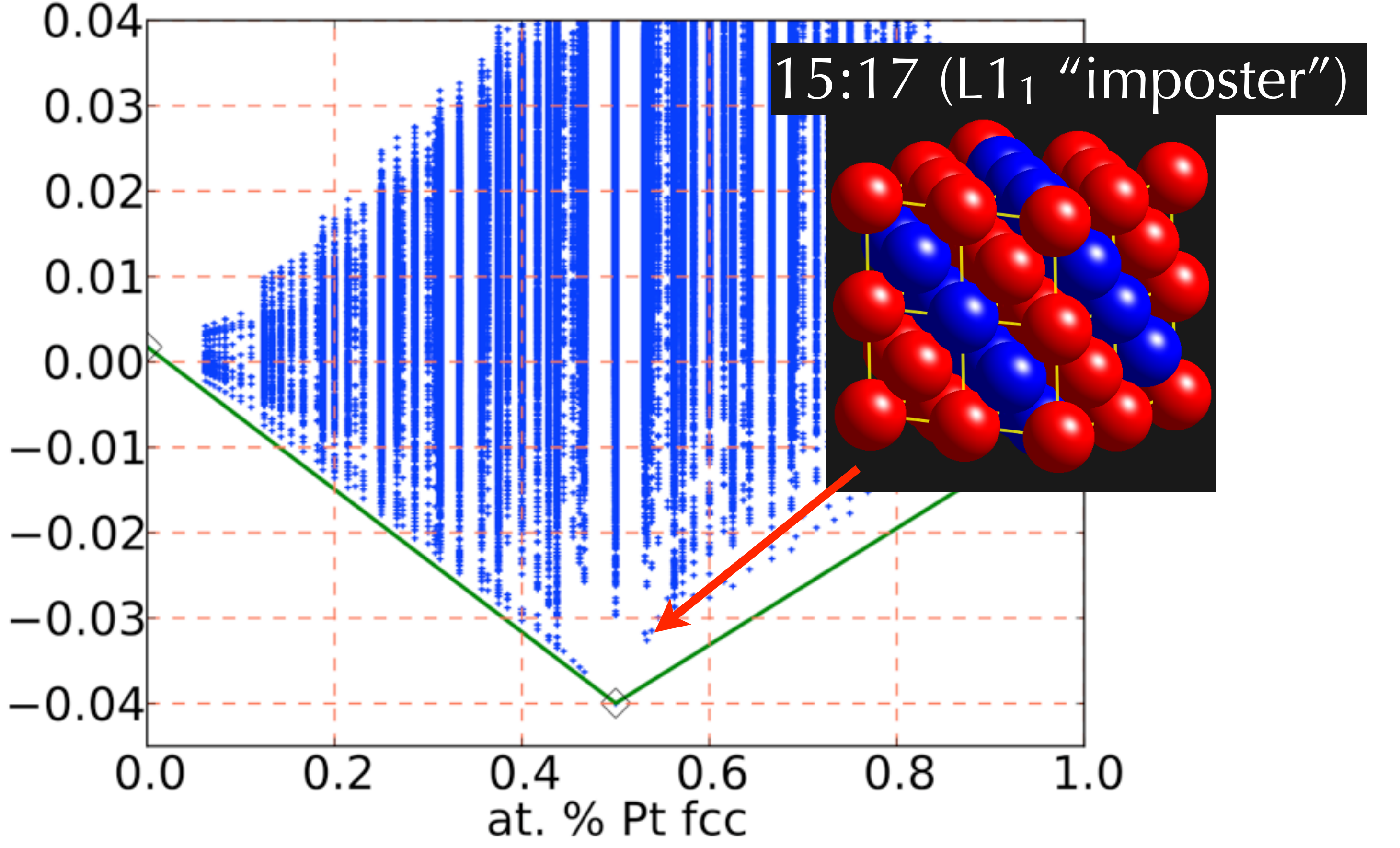

